\title{
On minimal requirements to obtain fuzzy concept-forming operators*
}

\author{
Juan Carlos Díaz ${ }^{1}$ Jesús Medina $^{1}$ Manuel Ojeda-Aciego ${ }^{2}$ \\ ${ }^{1}$ Department of Mathematics. University of Cádiz \\ Email: \{juancarlos.diaz, jesus.medina\}@uca.es \\ ${ }^{2}$ Dept. Matemática Aplicada, Universidad de Málaga. Spain \\ Email:aciego@uma.es
}

\begin{abstract}
In this work, we introduce sufficient and necessary conditions for a pair of fuzzy conjunctor and implication considered in a multi-adjoint frame under which the usual combinations of multi-adjoint sufficiency, possibility and necessity operators form either antitone or isotone Galois connections.
\end{abstract}

Keywords: Formal concept analysis; Galois connection; closure operators.

\section{Introduction}

Several extensions to the theory of formal concept analysis (FCA) have been introduced in the recent years, ranging from rough-set based approaches to possibility-theoretic approaches, and from interval valued contexts to similarity measures $[1,2,5,7,8,9,10,21]$. In addition, new types of incidence relations are being taken into account [13] together with alternative definitions of the concept-forming operators aimed at obtaining different interpretations for them. These operators are usually combined by pairs, since they form (either antitone or isotone) Galois connections, and, as a consequence, their compositions can be topologically interpreted as either closure or opening (kernel) operators.

In this work we are interested in fuzzy extensions of some of these previous approaches, particularly, the multi-adjoint framework $[15,16,17,18]$ since it embeds several other approaches $[3,4,12,14,20]$ which are based on residuated structures in order to build the concept-forming operators.

Recently, in [6] algebraic requirements were introduced under which the composition of conceptforming operators in four different versions (fuzzy sufficiency, possibility, necessity, and dual sufficiency) are either closure or opening operators.

Multi-adjoint concept lattices [18] are a convenient generalization of some existing approaches to fuzzy FCA which use several pairs of antitone Galois connections to build the concepts. More recently, both multi-adjoint property-oriented and

\footnotetext{
* Partially supported by the Spanish Science Ministry projects TIN2009-14562-C05 and TIN2012-39353-C04, and by Junta de Andalucía project P09-FQM-5233.
}

object-oriented concept lattices were introduced as a convenient generalization of fuzzy rough sets [15]; it is remarkable that in these settings, the main mathematical notion is that of isotone Galois connection.

The multi-adjoint frameworks cited above are founded on adjoint triples, which can be seen as convenient generalizations of the adjoint pair formed with classical conjunctor and implication connectives, or t-norms and their residua, and enable us to introduce alternative definitions of concept-forming operators in an $L$-fuzzy environment.

Following [6], we filter out some conditions originally imposed by adjoint triples which are not fundamental for the development of multi-adjoint FCA, in the sense that the Galois connections needed can be obtained by a smaller set of requirements. This applies both to antitone and isotone Galois connections. Sufficient and necessary conditions are introduced in order to obtain that the compositions of the concept-forming operators are either closure or opening operators. Consequently, a set of minimal requirements to build the different versions of multi-adjoint concept lattices are provided.

\section{Basic operators for multi-adjoint FCA}

Different generalizations of FCA coincide on the fact that the pair of fuzzy extensions of the crisp concept-forming operators is a Galois connection [11], for which there exist wo dual versions. We adopt in this section the standard one, in which both maps are order-reversing (antitone); these will be properly called Galois connections. The alternative version with the maps order-preserving, socalled isotone Galois connections, which will be studied later. We recall now the formal definition of (antitone) Galois connection.

Definition 1 Let $\left(P_{1}, \leq_{1}\right)$ and $\left(P_{2}, \leq_{2}\right)$ be posets, and $\downarrow: P_{1} \rightarrow P_{2},{ }^{\uparrow}: P_{2} \rightarrow P_{1}$ mappings, the pair $(\uparrow, \downarrow)$ forms an antitone Galois connection between $P_{1}$ and $P_{2}$ if and only if:

1. $\uparrow$ and $\downarrow$ are order-reversing.

2. $x \leq_{1} x^{\downarrow \uparrow}$ for all $x \in P_{1}$, that is, ${ }^{\downarrow \uparrow}$ is extensive (wrt $P_{1}$ )

3. $y \leq_{2} y^{\uparrow \downarrow}$ for all $y \in P_{2}$, that is, ${ }^{\uparrow \downarrow}$ is extensive (wrt $P_{2}$ ) 
From now on, we consider two complete lattices $\left(L_{1}, \preceq_{1}\right),\left(L_{2}, \preceq_{2}\right)$, a poset $(P, \leq)$ and two families of mappings $\swarrow^{i}: P \times L_{2} \rightarrow L_{1}, \nwarrow_{i}: P \times L_{1} \rightarrow L_{2}$, where $i$ belongs to an index set $\Lambda$.

We will also consider two sets $A$ and $B$ representing a set of attributes and a set of objects, respectively; together with a fuzzy relation between them, $R: A \times B \rightarrow P$. In addition, we also assume the existence of a mapping $\sigma: A \times B \rightarrow \Lambda$ relating each pair $(a, b)$ to a pair of implications $\left(\swarrow^{\sigma(a, b)}, \nwarrow_{\sigma(a, b)}\right)$, similarly to [18]. The tuple $(A, B, R, \sigma)$ will be called (formal) context.

Given a context $(A, B, R, \sigma)$, we define the oper$\operatorname{ators}^{\uparrow}: L_{2}^{B} \rightarrow L_{1}^{A}, \downarrow: L_{1}^{A} \rightarrow L_{2}^{B}$, as

$$
\begin{aligned}
& g^{\uparrow}(a)=\inf \left\{R(a, b) \swarrow^{\sigma(a, b)} g(b) \mid b \in B\right\} \\
& f^{\downarrow}(b)=\inf \left\{R(a, b) \nwarrow_{\sigma(a, b)} f(a) \mid a \in A\right\}
\end{aligned}
$$

for all $a \in A$ and $b \in B$.

We are slightly abusing notation in that $(\uparrow, \downarrow)$ depends on $\sigma$; furthermore, hereafter we will write $\swarrow^{a, b}, \nwarrow_{a, b}$ instead of $\swarrow^{\sigma(a, b)}, \nwarrow_{\sigma(a, b)}$.

We aim at finding a weaker setting under which the fuzzy extension of the crisp concept-forming operators still form an antitone Galois connection; i.e. we will introduce a sufficient and necessary condition in terms of the mappings $\swarrow^{i}, \nwarrow_{i}$, so that the operators $^{\uparrow}: L_{2}^{B} \rightarrow L_{1}^{A}, \downarrow: L_{1}^{A} \rightarrow L_{2}^{B}$ form an antitone Galois connection.

To begin with, we will prove first that these operators are antitone. The proposition below states that this is associated to the monotony of the operators $z \swarrow: L_{2} \rightarrow L_{1},{ }^{z \nwarrow}: L_{1} \rightarrow L_{2}$, where $z \in P$ and which are defined as $z \swarrow(y)=z \swarrow y,{ }^{z \nwarrow}(x)=$ $z \nwarrow x$, for all $y \in L_{2}, x \in L_{1}$.

Proposition 2 Mapping ${ }^{\uparrow}: L_{2}^{B} \rightarrow L_{1}^{A}$ is antitone for all formal context $(A, B, R, \sigma)$ if and only if $z \swarrow^{i}: L_{2} \rightarrow L_{1}$ is antitone for all $z \in P$ and $i \in \Lambda$.

Similarly, ${ }^{\downarrow}: L_{1}^{A} \rightarrow L_{2}^{B}$ is antitone for all formal context $(A, B, R, \sigma)$ if and only if $z \nwarrow_{i}: L_{1} \rightarrow L_{2}$ is antitone for all $z \in P$ and $i \in \Lambda$.

Remark 3 From the previous proposition, we can assume that, for all $i \in \Lambda$, arrows $\nwarrow_{i}, \swarrow^{i}$ are antitone in their second component.

The next result characterizes extensity of the composition $\uparrow \downarrow$ in terms of $\nwarrow_{i}, \swarrow^{i}$.

Theorem 4 Inequality $g \preceq_{2} g^{\uparrow \downarrow}$ holds for all context $(A, B, R, \sigma)$ and $g \in L_{2}^{B}$ if and only if the property below is satisfied $\forall y \in L_{2}, \forall z \in P, \forall i \in \Lambda$ :

$$
y \preceq_{2} z \nwarrow_{i}\left(z \swarrow^{i} y\right)
$$

The other one can be characterized similarly.

Theorem 5 Inequality $f \preceq_{1} f^{\downarrow \uparrow}$ holds for all context $(A, B, R, \sigma)$ and $f \in L_{1}^{A}$ if and only if the following property holds $\forall x \in L_{1}, \forall z \in P$ and $\forall i \in \Lambda$ :

$$
x \preceq_{1} z \swarrow^{i}\left(z \nwarrow_{i} x\right)
$$

Theorems 4 and 5, and Proposition 2 imply the following result.

Corollary 6 The pair $\left({ }^{\uparrow},{ }^{\downarrow}\right)$ forms an antitone $G a$ lois connection for all formal context $(A, B, R, \sigma)$ if and only if Properties $(F 1)$ and $(F 2)$ hold, and ${ }^{z \nwarrow_{i}}$, $z \swarrow^{i}$ are antitone for all $z \in P$ and $i \in \Lambda$.

Consider one pair of identical mappings, then our approach generalizes that of $[6$, Theorem 2], recalled in the following corollary.

Corollary 7 Given $(L, \preceq)=\left(L_{1}, \preceq_{1}\right)=\left(L_{2}, \preceq_{2}\right)$ and a pair of identical arrows $(\swarrow, \swarrow)$, then the compositions $\uparrow \downarrow, \downarrow \uparrow$ are closure operators if $\swarrow$ is antitone on its right argument and the following holds for all $y \in L, z \in P$ :

$$
y \preceq z \swarrow(z \swarrow y)
$$

As a consequence of Proposition 2, Theorems 4 and 5, the following characterization of pairs that form Galois connections is obtained.

Theorem 8 The pair $\left({ }^{\uparrow},{ }^{\downarrow}\right)$ is an antitone Galois connection, for all formal context $(A, B, R, \sigma)$, if and only if the pair $\left({ }_{z \swarrow} \swarrow^{i},{ }^{z \nwarrow_{i}}\right)$ is an antitone Galois connection, for all $z \in P$ and $i \in \Lambda$.

From the previous theorem, we can transform a problem between ${ }^{\uparrow}: L_{2}^{B} \rightarrow L_{1}^{A},{ }^{\downarrow}: L_{1}^{A} \rightarrow L_{2}^{B}$, whose definitions are complex, to a problem related to the more basic operators $\left(z \swarrow^{i},{ }^{z \nwarrow_{i}}\right)$.

It is worth to notice that the previous theorem does not imply a biunivocal correspondence between antitone Galois connections and pairs of implications associated with an adjoint triple, however we will keep on using the implication symbols as a vestige from our inspirational examples. Two operators $\phi$ and $\psi$ are introduced below, which allow to define an antitone Galois connection $(\uparrow, \downarrow)$; note, however, that $(\phi, \psi)$ is not a pair of residuated implications of an adjoint triple, particularly, because they are not fuzzy implications.

Example 9 Let us assume $\left(L_{1}, \preceq_{1}\right)=\left(L_{2}, \preceq_{2}\right)=$ $(P, \leq)=([0,1], \leq)$, where $\leq$ is the usual ordering in the unit interval, and the pair $(\phi, \psi)$, where $\phi:[0,1] \times[0,1] \rightarrow[0,1], \psi:[0,1] \times[0,1] \rightarrow[0,1]$ are defined as follows

$$
\begin{aligned}
& \phi(z, y)= \begin{cases}1 & \text { if } z \leq 1-y \\
\sqrt{\frac{1-y}{z}} & \text { if } 1-y<z<\frac{1}{2} \\
\frac{1-y}{z} & \text { if } 1-y<z, \quad z \geq \frac{1}{2}\end{cases} \\
& \psi(z, x)= \begin{cases}1-x^{2} z & \text { if } z<\frac{1}{2} \\
1-x z & \text { if } z \geq \frac{1}{2}\end{cases}
\end{aligned}
$$


Given $z \in[0,1]$, it will be shown that the pair of mappings $\phi_{z}:[0,1] \rightarrow[0,1], \psi_{z}:[0,1] \rightarrow[0,1]$ defined as $\phi_{z}(y)=\phi(z, y), \psi_{z}(x)=\psi(z, x)$, for all $x, y \in[0,1]$, form an antitone Galois connection.

First of all, from the definitions, we have that $\phi$ and $\psi$ are straightforwardly antitone in both arguments, hence $\phi_{z}$ and $\psi_{z}$ are antitone, in particular.

Now, we will prove that $x \leq \phi_{z}\left(\psi_{z}(x)\right)$ and $y \leq$ $\phi_{z}\left(\psi_{z}(y)\right)$, for all $x, y \in[0,1]$. Given $x, y, z \in[0,1]$ we have, by definition, that

$$
\phi(z, \psi(z, x))= \begin{cases}\phi\left(z, 1-x^{2} z\right) & \text { if } z<\frac{1}{2} \\ \phi(z, 1-x z) & \text { if } z \geq \frac{1}{2}\end{cases}
$$

If $z<\frac{1}{2}$, then $\phi\left(z, 1-x^{2} z\right)$ is equal to

$$
\begin{cases}1 & \text { if } z \leq 1-\left(1-x^{2} z\right) \\ \sqrt{\frac{1-\left(1-x^{2} z\right)}{z}} & \text { if } 1-\left(1-x^{2} z\right)<z\end{cases}
$$

which is equivalent to

$$
\begin{cases}1 & \text { if } z \leq x^{2} z \\ x & \text { if } x^{2} z<z\end{cases}
$$

consequently, in this case, $x \leq \phi_{z}\left(\psi_{z}(x)\right)$ holds.

$$
\begin{aligned}
& \text { If } z \geq \frac{1}{2}, \text { then } \\
& \phi(z, 1-x z)= \begin{cases}1 & \text { if } z \leq 1-\left(1-x^{2} z\right) \\
\frac{1-(1-x z)}{z} & \text { if } 1-(1-x z)<z\end{cases}
\end{aligned}
$$

similarly, $x \leq \phi_{z}\left(\psi_{z}(x)\right)$ holds.

For the other inequality, by definition, we obtain that $\psi(z, \phi(z, y))$ is:

$$
\begin{cases}\psi(z, 1) & \text { if } z \leq 1-y \\ \psi\left(z,\left(\sqrt{\frac{1-y}{z}}\right)\right) & \text { if } 1-y<z<\frac{1}{2} \\ \psi\left(z,\left(\frac{1-y}{z}\right)\right) & \text { if } 1-y<z, \quad z \geq \frac{1}{2}\end{cases}
$$

or equivalently

$$
\begin{cases}1-z & \text { if } z \leq 1-y \Leftrightarrow y \leq 1-z \\ 1-\left(\sqrt{\frac{1-y}{z}}\right)^{2} z & \text { if } 1-y<z<\frac{1}{2} \\ 1-\frac{1-y}{z} z & \text { if } 1-y<z, \quad z \geq \frac{1}{2}\end{cases}
$$

Thus, for all $y \in[0,1]$, we have $y \leq \psi(z, \phi(z, y))$.
Therefore, given $z \in[0,1]$, the pair $\left(\phi_{z}, \psi_{z}\right)$ is an antitone Galois connection and so, by Proposition 8, the mappings $\uparrow_{\phi}:[0,1]^{B} \rightarrow[0,1]^{A}, \downarrow^{\psi}:[0,1]^{A} \rightarrow$ $[0,1]^{B}$, defined as

$$
\begin{aligned}
& g^{\uparrow_{\phi}}(a)=\inf \{\phi(R(a, b), g(b)) \mid b \in B\} \\
& f^{\downarrow^{\psi}}(b)=\inf \{\psi(R(a, b), f(a)) \mid a \in A\}
\end{aligned}
$$

for all $a \in A$ and $b \in B$, form an antitone Galois connection.

Remark 10 From the example above, we have that the present framework is more general than the one given in [18], in which we considered adjoint triples $\left(\&_{i}, \swarrow^{i}, \nwarrow_{i}\right)$ and in the current setting we only need pairs $\left(\swarrow^{i}, \nwarrow_{i}\right)$, such that $\left(z \swarrow^{i}, z \nwarrow_{i}\right)$ are antitone Galois connections, for all $z \in P$.

Next example shows two fuzzy implications similar those used in fuzzy FCA and form an antitone Galois connection but, however, do not arise from an adjoint triple.

Example 11 Consider the pair $(\swarrow, \nwarrow)$ where $\swarrow:[0,1] \times[0,1] \rightarrow[0,1]$, and

$\nwarrow:[0,1] \times[0,1] \rightarrow[0,1]$ are defined by

$$
\begin{gathered}
z \nwarrow x= \begin{cases}1-x(1-z) & \text { if } z \leq \frac{1}{2} \\
1-x^{2}(1-z) & \text { if } z>\frac{1}{2}\end{cases} \\
z \swarrow y= \begin{cases}\frac{1-y}{1-z} & \text { if } z \leq \frac{1}{2}, \quad z<y \\
\sqrt{\frac{1-y}{1-z}} & \text { if } z>\frac{1}{2}, \quad z<y \\
1 \quad & \text { if } z \geq y\end{cases}
\end{gathered}
$$

These operators are similar to those in Example 9, in which we have replaced $z$ by $1-z$ and obtained, as a consequence, the monotonicity needed to obtain a fuzzy implication.

In this case, the pair $\left(z \swarrow,{ }^{z \nwarrow}\right)$ is an antitone $G a$ lois connection, for all $z \in[0,1]$, and it can be shown that there does not exist any conjunctor \& such that $(\&, \swarrow, \nwarrow)$ is an adjoint triple.

The examples above show that we are actually proposing in a more general framework than [18], but still we can define the set of all formal concepts $\langle g, f\rangle$, that is, the set

$\mathcal{M}=\left\{\langle g, f\rangle \mid g \in L_{2}^{B}, f \in L_{1}^{A}\right.$ and $\left.g^{\uparrow}=f, f^{\downarrow}=g\right\}$

with the ordering given by $\left\langle g_{1}, f_{1}\right\rangle \preceq\left\langle g_{2}, f_{2}\right\rangle$ if and only if $g_{1} \preceq_{2} g_{2}$ (equivalently $f_{2} \preceq_{1} f_{1}$ ), can be proven to be a complete lattice (the proof is similar to that in [18]).

We have just considered a more general framework on which the constructions are analogous, so it makes sense to keep the same names and still call the multi-adjoint concept lattice associated with a given formal context. 


\section{Multi-adjoint property-oriented and object-oriented concept lattices}

Inspired by [6], in this section we introduce sufficient and necessary conditions on the given fuzzy conjuntor and implication for the combinations of possibility and necessity operators to be isotone Galois connections. As a consequence, two kinds of concept lattices arise, generalizing the multi-adjoint object-oriented and property-oriented concept lattices given in [15].

Definition 12 Let $\left(P_{1}, \leq_{1}\right)$ and $\left(P_{2}, \leq_{2}\right)$ be posets, and $\downarrow: P_{1} \rightarrow P_{2},{ }^{\uparrow}: P_{2} \rightarrow P_{1}$ mappings, the pair $(\uparrow, \downarrow)$ forms an isotone Galois connection between $P_{1}$ and $P_{2}$ if and only if:

\section{1. $\uparrow$ and $\downarrow$ are order-preserving. \\ 2. $x^{\downarrow \uparrow} \leq_{1} x$, for all $x \in P_{1}$. \\ 3. $y \leq_{2} y^{\uparrow \downarrow}$, for all $y \in P_{2}$.}

In this setting, it is worth to recall the definition of dual order. Given a poset $(P, \leq)$, its dual ordering, $\leq^{\partial}$, is defined as $x_{1} \leq^{\partial} x_{2}$ if and only if $x_{2} \leq x_{1}$, for all $x_{1}, x_{2} \in P$. W will write $P$ instead of the partially ordered set $(P, \leq), P^{\partial}$ instead of $\left(P, \leq^{\partial}\right)$, and we will say that $P^{\partial}$ is the dual of $P$.

It is well known that the notion of isotone Galois connection follows from the original one simply by considering $P_{2}^{\partial}$ instead of $P_{2}$. Thus, an isotone Galois connection $\left({ }^{\uparrow}, \downarrow\right)$ on $P_{1}$ and $P_{2}$ is an antitone Galois connection on $P_{1}$ and $P_{2}^{\partial}$, and vice versa, and properties from antitone Galois connections can be translated to and from isotone Galois connections.

This idea has been recently used in [15] in order to relate several kinds of concept lattices. Similar transformations can be applied to use the properties obtained previously for the antitone Galois connection $\left({ }^{\uparrow}, \downarrow\right)$ in the framework of property-oriented and object-oriented concept lattices. Next, the details of this transformation for multi-adjoint propertyoriented concept lattices will be introduced. The object-oriented case is obtained analogously.

From now on, we will fix two complete lattices $\left(L_{2}, \preceq_{2}\right),\left(L_{3}, \preceq_{3}\right)$, a poset $(P, \leq)$ and two mappings \&: $P \times L_{2} \rightarrow L_{3}, \nwarrow: L_{3} \times P \rightarrow L_{2}$.

Given a context $(A, B, R, \sigma)$, we define two mappings $\uparrow_{\pi}: L_{2}^{B} \rightarrow L_{3}^{A}, \downarrow^{N}: L_{3}^{A} \rightarrow L_{2}^{B}$ as

$$
\begin{aligned}
& g^{\uparrow}(a)=\sup \left\{R(a, b) \&_{a, b} g(b) \mid b \in B\right\} \\
& f^{\downarrow^{N}}(b)=\inf \left\{f(a) \nwarrow_{a, b} R(a, b) \mid a \in A\right\}
\end{aligned}
$$

for each $g \in L_{2}^{B}, f \in L_{3}^{A}$ and $a \in A, b \in B$. These definitions are obvious generalizations of the classical possibility and necessity operators.

We establish here similar results to those in the previous section; but now, with respect to the operators $\uparrow_{\pi}, \downarrow_{N}$ and the underlying fuzzy conjunctors $\&_{i}$ and implications $\nwarrow_{i}$, with $i$ in the index set $\Lambda$.

To begin with, we focus on the monotony-related properties of these operators. Specifically, we consider operators ${ }_{x} \&: L_{2} \rightarrow L_{3}, \nwarrow_{x}: L_{3} \rightarrow L_{2}$, where $x \in P$ and which are defined as $x \&(y)=x \& y$, $\nwarrow_{x}(z)=z \nwarrow x$, for all $y \in L_{2}, z \in L_{3}$.

Lemma 13 Considering lattice $L_{3}^{\partial}$ instead of $L_{3}$, then the operators $\uparrow_{\pi}$ and $\downarrow^{N}$ satisfy Equations (1) and (2), with respect to \&, \op, respectively, where $\nwarrow_{o p}: P \times L_{3} \rightarrow L_{2}$ is defined as $x \nwarrow_{o p} z=z \nwarrow x$, for all $x \in P$ and $z \in L_{3}$.

Hence, the previous results can be transformed into this new approach. The following result, similar to that of Proposition 2, can be obtained:

Proposition 14 Mapping ${ }^{\uparrow \pi}: L_{2}^{B} \rightarrow L_{3}^{A}$ is isotone for all formal context $(A, B, R, \sigma)$ if and only if ${ }_{x} \&: L_{2} \rightarrow L_{3}$ is isotone for all $x \in P$.

Analogously, $\downarrow^{N}: L_{3}^{A} \rightarrow L_{2}^{B}$ is isotone for all formal context $(A, B, R, \sigma)$ if and only if $\nwarrow_{x}: L_{3} \rightarrow L_{2}$ is isotone for all $x \in P$.

Remark 15 In consequence, we can assume hereafter that conjunctors $\&_{i}$ are isotone in the right argument and arrows $\nwarrow_{i}$ are isotone in the left argument, for all $i \in \Lambda$.

The closure properties of the composition $\uparrow_{\pi} \downarrow^{N}: L_{2}^{B} \rightarrow L_{2}^{B}$ is characterized by using Lemma 13 and Theorem 4, which turns out to be a generalization of $[6$, Theorem 4].

Theorem 16 Inequality $g \preceq_{2} g^{\uparrow_{\pi} \downarrow^{N}}$ holds for all context $(A, B, R, \sigma)$ and $g \in L_{2}^{B}$ if and only if the following property holds $\forall x \in P, \forall y \in L_{2}, \forall i \in \Lambda$.

$$
y \preceq_{2}\left(x \&_{i} y\right) \nwarrow_{i} x
$$

Similarly, the interior property of $\downarrow^{N} \uparrow_{\pi}$ can be characterized by using Theorem 5 and Lemma 13 . This result is similar to [6, Theorem 5], but considering $\uparrow^{\uparrow}: L_{2}^{B} \rightarrow L_{3}^{A}, \downarrow^{N}: L_{3}^{A} \rightarrow L_{2}^{B}$ instead.

Theorem 17 Inequality $f^{\downarrow^{N} \uparrow_{\pi}} \preceq_{1} f$ holds for all context $(A, B, R, \sigma)$ and $f \in L_{1}^{A}$ if and only if the following property holds $\forall x \in P, \forall z \in L_{3}, \forall i \in \Lambda$ :

$$
x \&_{i}\left(z \nwarrow_{i} x\right) \preceq_{3} z
$$

Proposition 14, and Theorems 16 and 17, provide similar consequences to those above, but now concerning property-oriented concept lattices.

\section{Corollary 18}

1. Composition $\uparrow \downarrow^{N}: L_{2}^{B} \rightarrow L_{2}^{B}$ is a fuzzy closure operator for all context $(A, B, R, \sigma)$ if and only if $(P 1)$ is satisfied.

2. Composition $\downarrow^{{ }^{N} \uparrow_{\pi}}: L_{1}^{A} \rightarrow L_{1}^{A}$ is a fuzzy interior operator for all context $(A, B, R, \sigma)$ if and only if (P2) is satisfied.

Corollary 19 The pair $\left(\uparrow^{\uparrow}, \downarrow^{N}\right)$ is an isotone $G a$ lois connection for all formal context $(A, B, R, \sigma)$ if and only if $(P 1)$ and $(P 2)$ hold, and operators ${ }_{x} \&$ and $\nwarrow_{x}$ are isotone for all $x \in P$. 
As $\left({ }_{x} \&, \nwarrow_{x}\right)$ is isotone Galois connection for all $x \in P$ if and only if the mappings \& and $\nwarrow$ verify $(P 1),(P 2)$ and are isotone, we obtain the following:

Proposition 20 The pair $\left(\uparrow_{\pi}, \downarrow^{N}\right)$ is an isotone Galois connection for all context $(A, B, R, \sigma)$ if and only if the pair $\left({ }_{x} \&, \nwarrow_{x}\right)$ is an isotone Galois connection for all $x \in P$.

Hence, the properties of the pair $\left(\uparrow_{\pi}, \downarrow^{N}\right)$ depend just on \& and $\nwarrow$. Therefore, given two mappings $\&: P \times L_{2} \rightarrow L_{3}$ and $\nwarrow: L_{3} \times P \rightarrow L_{2}$, such that $\left({ }_{x} \&, \nwarrow_{x}\right)$ is isotone Galois connection for all $x \in P$, then the mappings ${ }^{\uparrow}: L_{2}^{B} \rightarrow L_{1}^{A}, \downarrow^{N}: L_{2}^{A} \rightarrow L_{1}^{B}$ allow to build a more general lattice than the concept lattice introduced in [15]. Specifically, the set

$\mathcal{M}_{\pi N}=\left\{\langle g, f\rangle \mid g \in L^{B}, f \in L^{A}, g^{\uparrow \pi}=f, f^{\downarrow^{N}}=g\right\}$

is a complete lattice, called multi-adjoint propertyoriented concept lattice, where the pairs of mappings $\langle g, f\rangle \in \mathcal{M}_{\pi N}$ are called multi-adjoint propertyoriented formal concepts.

Note, again, that the naming considered here for the concept lattice and for the concepts is exactly the same used in [15]. The reason is that, in spirit, they are equal. The main difference being that the considered operators can be more general, since the pair $(\&, \nwarrow)$ need not arise from an adjoint triple.

In the next example we introduce an isotone Galois connection $\left({ }_{x} \&, \nwarrow_{x}\right)$, although there does not exist $\swarrow$ such that $(\&, \swarrow, \nwarrow)$ is an adjoint triple. Moreover, by Proposition 20, we obtain that $\uparrow_{\pi}$ and $\downarrow^{N}$, defined as in Equations (3) and (4), form an isotone Galois connection and, then, a propertyoriented concept lattice can be considered.

Example 21 Consider $\left(L_{1}, \preceq_{1}\right)=\left(L_{2}, \preceq_{2}\right)=$ $(P, \leq)=([0,1], \leq)$, where $\leq$ is the usual ordering in the unit interval, and the mapping $\&:[0,1] \times[0,1] \rightarrow[0,1]$, defined $\forall x, y \in[0,1]$ as

$$
x \& y= \begin{cases}x \cdot y^{2} & \text { if } x<\frac{1}{2} \\ x \cdot y & \text { if } \frac{1}{2} \leq x\end{cases}
$$

Clearly, \& is isotone in both arguments.

Now, consider mappings $\swarrow:[0,1] \times[0,1] \rightarrow[0,1]$ and $\nwarrow:[0,1] \times[0,1] \rightarrow[0,1]$, defined, for all $x, y, z \in[0,1]$, as

$$
\begin{aligned}
& z \swarrow y=\sup \{x \mid x \& y \leq z\} \\
& z \nwarrow x=\sup \{y \mid x \& y \leq z\}
\end{aligned}
$$

If in these definitions, the supremum is a maximum, then the mappings are residuated implications associated with \&.

We will prove first that the supremum in the definition of $\nwarrow$ is indeed a maximum, that is,

$$
z \nwarrow x=\sup \{y \mid x \& y \leq z\}=\max \{y \mid x \& y \leq z\}
$$

For that, given $x, z \in[0,1]$, we compute the supremum of the values $Y=\{y \mid x \& y \leq z\}$ and we check whether they are or are not in $Y$. We reason by cases:

- If $x \leq z$, then $x \& y \leq x \& 1=x \leq z$ and so $\sup Y=\sup \{y \mid x \& y \leq z\}=1$, which is in $Y$.

- If $z<x<\frac{1}{2}$, then $x \& \sqrt{\frac{z}{x}}=x \cdot\left(\sqrt{\frac{z}{x}}\right)^{2}=$ $x \cdot \frac{z}{x}=z$. Moreover, if we consider an element $y \in[0,1]$, such that $\sqrt{\frac{z}{x}}<y$ then

$$
z=x \cdot \frac{z}{x}=x \cdot\left(\sqrt{\frac{z}{x}}\right)^{2}<x \cdot y^{2}=x \& y
$$

Therefore, $\sup Y=\sup \{y \mid x \& y \leq z\}=\sqrt{\frac{z}{x}}$, which is in $Y$.

- If $z<x$ and $\frac{1}{2} \leq x$, then $x \& \frac{z}{x}=x \cdot \frac{z}{x}=z$. Moreover, if we assume an element $y \stackrel{x}{\in}[0,1]$, such that $\frac{z}{x}<y$, then

$$
z=x \cdot \frac{z}{x}<x \cdot y=x \& y
$$

Therefore, $\sup Y=\sup \{y \mid x \& y \leq z\}=\frac{z}{x}$, which is in $Y$.

Consequently, we obtain that

$$
\sup \{y \mid x \& y \leq z\}=\max \{y \mid x \& y \leq z\}
$$

On the other hand, we will prove that this conclusion cannot be obtained for the other implication. We will denote $\{x \mid x \& y \leq z\}$ as $X$.

- If $y \leq z$, then $x \& y \leq 1 \& y=y \leq z$ and so $\sup X=\sup \{x \mid x \& y \leq z\}=1$, which is an element of $X$.

- If $z<y \leq 2 z$, then $\frac{1}{2} \leq \frac{z}{y}$ and, therefore, $\frac{z}{y} \& y=\frac{z}{y} \cdot y=z$. Moreover, if $\frac{1}{2} \leq \frac{z}{y}<x$ then $z=\frac{z}{y} \cdot y<x \cdot y=x \& y$. Hence, $\sup \{x \mid$ $x \& y \leq z\}=\frac{z}{y}$, which is in $X$.

- If $\sqrt{2 z}<y$, then $\frac{z}{y^{2}}<\frac{1}{2}$ and so $\frac{z}{y^{2}} \& y=$ $\frac{z}{y^{2}} \cdot y^{2}=z$. If we consider $x \in[0,1]$ such that $\frac{z}{y^{2}}<x$, then we obtain the following two inequalities:

$$
\begin{array}{r}
z=\frac{z}{y^{2}} \cdot y^{2}<x \cdot y^{2} \\
z \leq \frac{z}{y}=\frac{z}{y^{2}} \cdot y<x \cdot y
\end{array}
$$

As a consequence, independently of the value of $x$, we obtain $z<x \& y$. Thus, $\sup X=\frac{z}{y^{2}}$, which is an element of $X$. 
- If $2 z<y \leq \sqrt{2 z}$, then for all $x<\frac{1}{2}, x \& y=$ $x \cdot y^{2}<\frac{1}{2} \cdot 2 z=z$ and $z<\frac{y}{2}=\frac{1}{2} \cdot y=\frac{1}{2} \& y$. Hence, $\sup X=\sup \{x \mid x \& y \leq z\}=\sup \{x \in$ $\left.\left[0, \frac{1}{2}\right)\right\}=\frac{1}{2}$, however, $\frac{1}{2} \& y \not \leq z$, that is $\frac{1}{2} \notin X$ and the supremum is not a maximum.

Therefore, in this case we obtain that $\sup X \neq$ $\max X$. Consequently, $(\&, \swarrow, \nwarrow)$ is not an adjoint triple, although $\left(x \&, \nwarrow_{x}\right)$ is an isotone Galois connection, for all $x \in[0,1]$.

Finally, the expressions for the implications are:

$$
\begin{aligned}
z \nwarrow x & =\max \{y \mid x \& y \leq z\} \\
& = \begin{cases}1 & \text { if } x \leq z \\
\sqrt{\frac{z}{x}} & \text { if } z<x<\frac{1}{2} \\
\frac{z}{x} & \text { if } z<x, \quad x \geq \frac{1}{2}\end{cases} \\
z \swarrow y & =\sup \{x \mid x \& y \leq z\} \\
& = \begin{cases}\frac{1}{z} & \text { if } y \leq z \\
\frac{1}{y} & \text { if } z<y \leq 2 z \\
\frac{1}{2} & \text { if } 2 z<y \leq \sqrt{2 z} \\
\frac{z}{y^{2}} & \text { if } \sqrt{2 z}<y\end{cases}
\end{aligned}
$$

Operators as those used above are not just pathological operators, they could be useful to distinguish between the values provided by the relation and those given by the fuzzy subset of object, and this is interesting for applications. For instance, the conjunctor in the example simply weights similarly the values given by the relation and the fuzzy subset when the former is greater or equal than the threshold $1 / 2$.

\section{Conclusions}

Characterizations of different Galois connections arising in FCA have been introduced, in such a way that the necessary algebraic requirements are translated directly on the fuzzy conjunctors and implications considered in the underlying multi-adjoint context.

As a consequence, it turns out that the approach in $[15,18]$ is applicable in more general situations, in particular, the conjunctors and implications in the multi-adjoint formal context need not form adjoint triples, but satisfy weaker conditions instead.

\section{References}

[1] C. Alcalde, A. Burusco, R. Fuentes-González, and I. Zubia. The use of linguistic variables and fuzzy propositions in the L-fuzzy concept theory. Computers $\& 3$ Mathematics with Applications, 62(8):3111-3122, 2011.
[2] F. Alqadah and R. Bhatnagar. Similarity measures in formal concept analysis. Annals of Mathematics and Artificial Intelligence, 61(3):245-256, 2011.

[3] R. Bělohlávek. Fuzzy concepts and conceptual structures: induced similarities. In Joint Conference on Information Sciences, pages 179-182, 1998.

[4] A. Burusco and R. Fuentes-González. The study of $L$-fuzzy concept lattice. Mathware $\&$ Soft Computing, 3:209-218, 1994.

[5] Y. Chen and Y. Yao. A multiview approach for intelligent data analysis based on data operators. Information Sciences, 178(1):1-20, 2008.

[6] Y. Djouadi and H. Prade. Possibility-theoretic extension of derivation operators in formal concept analysis over fuzzy lattices. Fuzzy Optimization and Decision Making, 4:287-309, 2011.

[7] D. Dubois and H. Prade. On possibility theory, formal concept analysis and granulation: Survey. $A p$ plied and Computational Mathematics, 10(1):1019, 2011.

[8] D. Dubois and H. Prade. Possibility theory and formal concept analysis: Characterizing independent sub-contexts. Fuzzy Sets and Systems, 196:4-16, 2012 .

[9] I. Düntsch and G. Gediga. Approximation operators in qualitative data analysis. In Theory and Applications of Relational Structures as Knowledge Instruments, pages 214-230, 2003.

[10] A. Formica. Semantic web search based on rough sets and fuzzy formal concept analysis. KnowledgeBased Systems, 26:40-47, 2012.

[11] B. Ganter and R. Wille. Formal Concept Analysis: Mathematical Foundation. Springer Verlag, 1999.

[12] G. Georgescu and A. Popescu. Non-dual fuzzy connections. Arch. Math. Log., 43(8):1009-1039, 2004.

[13] L. Guo, F. Huang, Q. Li, and G. Zhang. Power contexts and their concept lattices. Discrete Mathematics, 311(18-19):2049-2063, 2011.

[14] H. Lai and D. Zhang. Concept lattices of fuzzy contexts: Formal concept analysis vs. rough set theory. International Journal of Approximate Reasoning, 50(5):695-707, 2009.

[15] J. Medina. Multi-adjoint property-oriented and object-oriented concept lattices. Information Sciences, 190:95-106, 2012.

[16] J. Medina and M. Ojeda-Aciego. Multi-adjoint tconcept lattices. Information Sciences, 180(5):712725, 2010.

[17] J. Medina and M. Ojeda-Aciego. On multi-adjoint concept lattices based on heterogeneous conjunctors. Fuzzy Sets and Systems, 208: 95-110 (2012)

[18] J. Medina, M. Ojeda-Aciego, and J. Ruiz-Calviño. Formal concept analysis via multi-adjoint concept lattices. Fuzzy Sets and Systems, 160(2):130-144, 2009.

[19] J. Medina, M. Ojeda-Aciego, A. Valverde, and P. Vojtáš. Towards biresiduated multi-adjoint logic programming. Lect. Notes in Artificial Intelligence, 3040:608-617, 2004.

[20] S. Pollandt. Fuzzy Begriffe. Springer, Berlin, 1997.

[21] Y. Y. Yao. A comparative study of formal concept analysis and rough set theory in data analysis. Lecture Notes in Artificial Intelligence, 3066:59-68, 2004. 